\title{
A TEST OF THE INFLUENCE OF ISOALLELIC VARIATION UPON A QUANTITATIVE CHARACTER
}

\author{
JAMES N. THOMPSON, Jr* \\ Department of Genetics, University of Cambridge, Milton Road, Cambridge CB4 IXH
}

Received 23.v.75

\section{Summary}

\begin{abstract}
In order to test the assumption that isoalleles can contribute to polygenic variation, several isoalleles of cubitus interruptus were examined for their influence upon the quantitative expression of a related venation mutant, veinlet. In spite of the fact that veinlet and cubitus interruptus affect closely related developmental processes, the $c i$ isoalleles had no effect upon the expression of veinlet. This test does not, of course, disprove the hypothesis that isoalleles play a part in polygenic variation. It is clear, however, that not all seemingly relevant isoalleles are necessarily effective elements in a given polygenic system.
\end{abstract}

\section{InTRODUCTION}

A GLEAR understanding of selection responses requires that we know what kinds of gene effects are included in the general category "polygenic variation". It has been obvious for a long time that the magnitudes of individual loci affecting quantitative characters can vary widely. Even major gene mutations can contribute to selection responses as Waddington (1956, 1961) and Bateman (1959) found in experiments designed to study genetic assimilation of bithorax and dumpy phenocopies in Drosophila melanogaster.

In general, however, polygenes have effects which are small relative to the effects of uncontrolled environmental variation (Mather, 1943). In addition to polygenes having small, general effects upon a particular character (Thompson, 1973, 1974), mutant-specific polygene effects have been reported by Scharloo (1964), Fraser (1967), Rendel (1968), and others. Such locusspecific polygenes might reflect variation in the efficiency of control genes or similar loci having direct influence upon a particular locus.

It is widely assumed that a significant proportion of polygenic variation is due to mutations producing minor modifications at loci which are also known through major gene mutations. Isoalleles have been defined (Stern and Schaeffer, 1943) as different alleles which are indistinguishable except when special tests are used. Although Waddington, Graber, and Woolf (1957) failed to find mutations to isoalleles of cubitus interruptus Wallace during selection in an isogenic line, most experimental designs cannot exclude the possibility that such mutations are an important component of polygenic variation. Indeed, isoalleles have been implicated in a number of selection experiments (e.g. det in Milkman, 1970b), and systems such as eye

* Present address: Department of Zoology, University of Oklahoma, 730 Van Vleet Oval, Norman, Oklahoma 73069 U.S.A. 
colour in Drosophila (Green, 1959) show how isoallelic variation could function in affecting a quantitative character.

But saying that it is likely that polygenic variation includes isoalleles is not the same as demonstrating that this is true. Unfortunately, there are major limitations to any attempt to demonstrate isoalleles in a selection line. Typical polygene location analyses (Thoday, 1961) associate a particular small chromosome region with a measurable phenotypic effect. But the small magnitude of phenotypic changes produced by individual polygenic loci means that it is seldom possible to locate them precisely or determine whether they are single genetic loci or a complex of closely linked loci producing similar changes in the phenotype (McMillan and Robertson, 1974; Thompson and Thoday, 1974). The precision of the map position is not sufficient by itself to demonstrate allelism. Except in special systems (Milkman, 1970a), direct allelism tests require that both the polygenic locus and the gene to be tested are on identical isogenic backgrounds. Direct allelism tests are, therefore, seldom practical, and one is always left with the possibility that a closely linked locus is in fact producing the effects attributed to the "isoallele".

The most obvious way around these limitations is to approach the problem from the opposite direction. Rather than asking whether a particular selection response involves isoalleles of known major gene loci, one can ask whether known isoalleles are able to affect the expression of a particular quantitative character. To this end an attempt has been made to determine whether known cubitus interruptus isoalleles have any effect upon quantitative variation in the length of the L4 vein in flies carrying the mutant veinlet.

One can identify two classes of "wild type" isoalleles at the cubitus interruptus (ci) locus: those which are completely dominant over $c i$ in heterozygous condition and those in which some penetrance of the L4 vein gap phenotype of $c i$ occurs in heterozygotes (Hochman, 1958).

The effect of such $c i$ isoalleles upon veinlet (ve) expression was chosen as a test system for several reasons. First, a number of $c i$ isoalleles have been studied in detail and some are available from culture collections (Lindsley and Grell, 1967). In addition, the $c i$ locus is on the fourth chromosome, and control chromosomes or chromosomes carrying an isoallele to be tested can, therefore, be substituted into a marked stock of veinlet, a third chromosome mutant, without otherwise disturbing the inbred genetic background.

Most important, however, $c i$ and ve not only interact superadditively (Thompson, 1975), but some of the polygenic loci which act as L4 vein length modifiers of veinlet also affect the expression of cubitus interruptus in the same way (Thompson, 1973). This strongly suggests that the two loci act upon closely related processes in the development of venation and it is, therefore, reasonable to expect isoalleles of one to modify the expression of the other. Vein length can be measured accurately in large population samples, so that even small effects should be detectable.

\section{Methods}

Two isoalleles $\left(c i^{+3}\right.$ and $c i^{+5}$, hereafter abbreviated $+^{3}$ and $+^{5}$ ) were kindly supplied by Dr B. Hochman. Two fourth chromosomes used as controls were obtained from two separate iso-female lines from Oklahoma (abbreviated $+^{01}$ and $+^{02}$ ). In $\mathrm{F}_{1}$ heterozygotes from crosses between 
inbred $c i$ ey ${ }^{R}$ and these four chromosomes, $+{ }^{3}$ and $+{ }^{5}$ produced a large proportion of flies having L4 vein gaps while all offspring from $+{ }^{01}$ and $+{ }^{02}$ were wild type (table 1 ).

The breeding programme used to substitute an isoallele or control chromosome into the inbred veinlet genome was derived from the standard whole chromosome assay programme which capitalises upon the fact that there is usually no crossing over in male Drosophila melanogaster. Tested and standard chromosomes are inherited intact. An inbred bw; ve; $e y^{2}$ stock was used as the standard (bw, brown, chrom. 2; ey ${ }^{2}$, eyeless ${ }^{2}$, chrom. 4).

TABLE 1

Frequency of L4 vein gaps in flies heterozygous for the ci ey " standard" fourth chromosome and for one of the tested chromosomes carrying a cubitus interruptus isoallele $†$

\begin{tabular}{|c|c|c|c|c|}
\hline \multirow[b]{2}{*}{$c i$ isoallele } & \multicolumn{2}{|c|}{ Flies having L4 gaps } & \multicolumn{2}{|c|}{ Frequency } \\
\hline & Females & Males & Females & Males \\
\hline$+^{3}$ & $79(110)$ & $66(69)$ & 0.718 & 0.957 \\
\hline+5 & $118(172)$ & $129(134)$ & $0 \cdot 686$ & 0.963 \\
\hline+01 & $0(117)$ & $0(91)$ & 0 & 0 \\
\hline+02 & $0(84)$ & $0(74)$ & 0 & 0 \\
\hline
\end{tabular}

First, virgin $b w ; v e ; e y^{2}$ females are mated to males carrying the fourth chromosome to be tested. $F_{1}$ male heterozygotes are then backcrossed singly to several $b w ; v e ; e y^{2}$ females. The progeny of each single backcrossed male provide one of the replicates for each of the chromosomes tested ( $N=180$ flies). In the backcross progeny, flies homozygous for bw and for ve were separated from the others. These $b w ; v e$ flies are homozygous for the inbred standard background except for chromosome 4 and are of two types: those homozygous for $e y^{2}$ (control class) and those heterozygous for $e y^{2}$ and for the tested fourth chromosome (experimental class). Only heterozygous effects were measured, since only segregating heterogeneity will contribute significantly to the genetic component of variation. The mutant $e y^{2}$ does not affect vein length (Stern, 1943).

L4 vein lengths were measured in three flies of each sex of each genotype in all replicates. Variations in fly size were standardised by expressing all vein lengths as the sine transformed ratio of L4 vein length to total wing length (Thompson, 1974).

\section{Results AND Discussion}

Mean vein lengths and summaries of the resulting analyses of variance are given in tables 2 and 3 . Several things are clear from these results. The most important observation is that none of the chromosomes causes a significant decrease in the length of the L4 vein in veinlet, as would have been expected if the $c i$ isoalleles had been acting as "short vein polygenes" in the veinlet background. In only one case was a significant difference between experimental and control fourth chromosomes observed, i.e. $+{ }^{3}$, but from table 2 it can be seen that this was due to an increase, rather than a decrease, in L4 vein length. 
In addition, there is significant variance associated with sexes as is typical of vein length phenotypes, and there is some variation between replicates. No higher order interaction terms are significant.

There was every reason to expect the $+^{3}$ and $+t^{5}$ isoalleles to be effective modifiers of $\mathrm{L} 4$ vein length in veinlet. Both $c i$ and ve are affected by polygenic variation and respond readily to artificial selection. Indeed, many of the

\section{TABLE 2}

Mean relative vein lengths of the $L 4$ veins in control and experimental veinlet fies. (IVc, control; IV $V^{e}$, experimental chromosome carrying a tested isoallele). Only heterozygous isoallele effects are measured $\dagger$

\begin{tabular}{|c|c|c|c|c|}
\hline \multirow[b]{2}{*}{ Isoallele } & \multicolumn{2}{|c|}{$\begin{array}{c}\text { IVe } / \mathrm{IV}^{\mathrm{c}} \\
\text { (experimental) }\end{array}$} & \multicolumn{2}{|c|}{$\begin{array}{l}\text { IVe/IVe } \\
\text { (control) }\end{array}$} \\
\hline & Females & Males & Females & Males \\
\hline$t^{3}$ & 0.584 & 0.565 & 0.565 & 0.559 \\
\hline$t^{5}$ & 0.570 & 0.556 & 0.563 & 0.555 \\
\hline$+{ }^{01}$ & 0.547 & $0 \cdot 540$ & 0.538 & 0.535 \\
\hline$+{ }^{02}$ & 0.569 & 0.550 & 0.564 & 0.551 \\
\hline
\end{tabular}

$\dagger$ Lengths are expressed as the mean sine transformed ratio of vein length to total wing length. $N=180$ in each of the four sets of chromosomes tested; 15 replicate cultures sampled in each analysis.

TABLE 3

Summary of the analyses of variance comparing the length of the L4 veins in veinlet flies with and without one of the ci isoalleles. Significance levels $\dagger$ are from the four analyses of variance, each item having been tested against its respective residual mean square for individual flies. Testing items against appropriate interaction terms does not alter the conclusions drawn from the table

\section{Item}

Difference between chromosomes

Difference between sexes

Variation between replicates

Other terms (interactions)

\begin{tabular}{cccc}
\multicolumn{4}{c}{ Isoallele } \\
$\overbrace{}^{3}$ & $+^{8}$ & $+^{01}$ & $+^{02}$ \\
$* * *$ & n.s. & n.s. & n.s. \\
$* * *$ & $* *$ & n.s. & $* * *$ \\
$* * *$ & $*$ & $*$ & $* *$ \\
n.s. & n.s. & n.s. & n.s.
\end{tabular}

$$
\text { †* } 0.05>P>0.01 ; * * 0.01>P>0.001 ; * * * P<0.001 \text {. }
$$

polygenic loci which modify L4 vein expression act upon both ci and ve, indicating that the two mutants probably affect closely related developmental steps. The major mutants themselves interact superadditively as homozygotes. This close developmental relationship seemed to make the system optimal for the detection of isoallelic effects.

This test is, of course, only one of an almost infinite series of similar experiments which one might do with different isoallele sets and different characters. The $c i$ isoalleles have one major advantage, however. Since most of the fourth chromosome genes in Drosophila melanogaster are known (Hochman, 1973), one can reasonably say that $c i$, with the associated $c i^{D}$ locus, is the only region which has a marked effect upon L4 vein length, though theoretically one of the lethals might also represent a locus which in the wild type individual affects vein length. Thus, it is unlikely that linked 
loci would be responsible for any observed phenotypic effect associated with an observed isoallelic difference. The negative results are likewise unlikely to be a consequence of alleles linked to the $c i$ isoaileles but having the opposite phenotypic effect.

It should not be argued that, on the basis of these results, isoalleles do not contribute to polygenic variation. It is quite likely that isoalleles are a major component of such variation. Scharloo (1970), for example, discovered genes having fairly large, though opposite, effects upon wing vein length in selected lines of the mutant cubitus interruptus Dominant of Gloor $\left(c i^{D-G}\right)$. These vein length modifiers were tightly-linked and arranged in a balanced suppressor-enhancer relationship, so that no phenotypic effect was measurable until the linkage was broken by recombination. Mapping of these modifiers showed them to be alleles at two loci also known through major gene mutations, crossveinless- 2 and plexus. In addition, although I know of no association of an electrophoretic variant with a phenotypic change, there is some evidence that selective differences exist between electrophoretic isoalleles at enzyme loci (see Wills et al., 1975, and references there). It is, therefore, quite possible that these electrophoretic isoalleles might contribute to polygenic variation in some character.

The conclusion which can be drawn from this study is not, therefore, that isoalleles play no part in polygenic variation, but rather that the relationship between isoalleles and polygenic variation is not always obvious. Certainly thresholds might produce situations in which a limited set of isoalleles or interactions involving only particular loci were effective, and indeed other $c i$ isoalleles might be effective veinlet expression modifiers. But it is at least clear that not all seemingly relevant isoalleles are necessarily effective elements in a given polygenic system.

Acknowledgments. - I am grateful to Professor J. M. Thoday for stimulating discussions and for comments on this manuscript. This work was supported by a Science Research Council grant to Professor Thoday.

\section{References}

Bateman, к. G. 1959. The genetic assimilation of the dumpy phenocopy. 7. Genet., 56, 341-352.

FRASER, A. 1967. Variation of scutellar bristles in Drosophila. XV. Systems of modifiers. Genetics, 57, 919-934.

GREEN, M. M. 1959. The discrimination of wild-type isoalleles at the white locus of Drosophila melanogaster. Proc. Natl. Acad. Sci., U.S.A., 45, 549-553.

hоснмаN, в. 1958. Competition between wild type isoalleles in experimental populations of Drosophila melanogaster. Genetics, 43, 101-121.

носнмan, в. 1973. Analysis of a whole chromosome in Drosophila. Cold Spring Harbor Symp. Quant. Biol., 38, 581-589.

LINDSLEY, D. L., AND GRELL, E. H. 1967. Genetic Variations of Drosophila melanogaster. Carnegie Institution of Washington Publ. No. 627.

MAther, K. 1943. Polygenic inheritance and natural selection. Biol. Rev., 18, 32-64.

MCMILLAN, I., AND ROBERTSON, A. 1974. The power of methods for the detection of major genes affecting quantitative characters. Heredity, 32, 349-356.

MrLkman, R. D. 1970a. The genetic basis of natural variation. X. Recurrence of cve polygenes. Genetics, 65, 289-303.

milkman, R. 1970b. The genetic basis of natural variation in Drosophila melanogaster. Adv. Genet., 15, 55-114.

RENDEL, J. M. 1968. The control of developmental processes. In Evolution and Environment (ed. E. T. Drake), 341-349. Yale University Press, New Haven. 
SCHARLOo, w. 1964. Mutant expression and canalization. Nature, 203, 1095-1096.

sCHARLOo, w. 1970. Stabilizing and disruptive selection on a mutant character in Drosophila. II. Polymorphism caused by a genetical switch mechanism. Genetics, 65, 681-691. STERN, c. 1943. Genic action as studied by means of the effects of different doses and combinations of alleles. Genetics, 28, 441-475.

STERN, C., AND SCHAEFFER, E. w. 1943. On wild-type iso-alleles in Drosophila melanogaster. Proc. Natl. Acad. Sci., U.S.A., 29, 361-367.

THODAY, J. M. 1961. Location of polygenes. Nature, 191, 368-370.

тномРSON, J. N., JR. 1973. General and specific effects of modifiers of mutant expression. Genet. Res., Camb., 22, 211-215.

THOMPSON, J. N., JR. 1974. Studies on the nature and function of polygenic loci in Drosophila. I. Comparison of genomes from selection lines. Heredity, 33, 373-387.

THOMPSON, J. N., JR. 1975. Region-specific interactions in venation mutants. Dros. Inf. Serv. (in press).

THOMPSON, J. N., JR., AND THODAY, J. M. 1974. A definition and standard nomenclature for "polygenic loci ". Heredity, 33, 430-437.

WADDINGTON, с. H. 1956. Genetic assimilation of the bithorax phenotype. Evolution, 10, $1-13$.

waddington, с. н. 1961. Genetic assimilation. Adv. Genet., 10, 257-293.

WADDINGTON, C. H., GRABER, H., AND WOOLF, B. 1957. Iso-alleles and the response to selection. 7. Genet., 55, 246-250.

witls, C., PHELPS, J., AND FERGuson, R. 1975. Further evidence for selective differences between isoalleles in Drosophila. Genetics, 79, 127-141. 